HERBERT H. UHLIG

Korrosion und Korrosionsschutz 
Titel des Originalwerkes:

\section{Corrosion and Corrosion Control}

An Introduction to Corrosion

Science and Engineering

Second Edition

by

Herbert H. UHLIG

Professor of Metallurgie In Charge of

Corrosion Laboratory

Massachusetts Institute of Technology

Authorized translation from English language

edition published by John Wiley \& Sons, Inc., New York Copyright (c) 1963, 1971 by John Wiley \& Sons, Inc.

All Right Reserved 


\section{KORROSION \\ UND \\ KORROSIONSSCHUTZ}

von

HERBERT H. UHLIG

Herausgegeben von

Prof. em. Dr.-Ing. habil., Dr. rer. nat. mult. h. c. Kurt Schwabe

Übersetzt und ergänzt von

Dr. Werner Schmidt

Mit 129 Abbildungen und 34 Tabellen

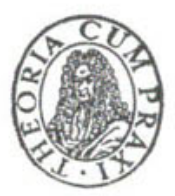

AKADE M I E - VERLAG - B ERLIN 1975 
2., bearbeitete Auflage

Erschienen im Akademie-Verlag, 108 Berlin. Leipziger Str. 3-4

Von John Wiley \& Sons, Inc., New York.

genehmigte Lizenzausgabe

Copyright (e) 1963, 1971 by John Wiley \& Sons, Inc., New York

Lizenznummer: $202 \cdot 100 / 487 / 75$

Gesamtherstellung: VEB Druckhaus „Maxim Gorki“", 74 Altenburg

Bestellnummer: 7612581 (5647) - LSV 3015/1205

Printed in GDR

EVP 58,- 